\title{
Changes in brain morphology in patients with obstructive sleep apnoea
}

\author{
M J Morrell, ${ }^{1,2}$ M L Jackson, ${ }^{3,4} \mathrm{G}$ L Twigg, ${ }^{1}$ R Ghiassi, ${ }^{5} \mathrm{D}$ W McRobbie, ${ }^{6}$ R A Quest, ${ }^{6}$ \\ H Pardoe, ${ }^{7}$ G S Pell, ${ }^{7}$ D F Abbott, ${ }^{7}$ P D Rochford, ${ }^{7}$ G D Jackson, ${ }^{7}$ R J Pierce, ${ }^{4}$ \\ F J O'Donoghue, ${ }^{7,4}$ D R Corfield ${ }^{8}$
}

\begin{abstract}
${ }^{1}$ National Heart and Lung Institute, Imperial College, London, UK

${ }^{2}$ Biomedical Research Unit at the Royal Brompton and Harefield NHS Foundation Trust, London, UK

${ }^{3}$ Sleep and Performance Research Center, Washington State University, Spokane, Washington, USA

${ }^{4}$ Institute for Breathing and Sleep, Austin Health,

Melbourne, Australia

${ }^{5}$ Respiratory Medicine, Charing

Cross Hospital, London, UK

${ }^{6}$ Radiological Sciences Unit, Imperial College, London, UK

${ }^{7}$ Brain Research Institute, Florey Neuroscience Institutes

(Austin), Melbourne, Australia

${ }^{8}$ Institute for Science and

Technology in Medicine, Keele University, Staffordshire, UK
\end{abstract}

\section{Correspondence to}

Mary J Morrell, Clinical and

Academic Unit of Sleep and Breathing, Royal Brompton

Hospital, Sydney Street, London

SW6 3NP, UK:

m.morrell@imperial.ac.uk

Received 7 September 2009 Accepted 26 June 2010

\begin{abstract}
Background Obstructive sleep apnoea (OSA) is a common disease that leads to daytime sleepiness and cognitive impairment. Attempts to investigate changes in brain morphology that may underlie these impairments have led to conflicting conclusions. This study was undertaken to aim to resolve this confusion, and determine whether OSA is associated with changes in brain morphology in a large group of patients with OSA, using improved voxel-based morphometry analysis, an automated unbiased method of detecting local changes in brain structure.
\end{abstract}

Methods 60 patients with OSA (mean apnoea hypopnoea index 55 (95\% Cl 48 to 62) events/h, 3 women) and 60 non-apnoeic controls (mean apnoea hypopnoea index 4 (95\% $\mathrm{Cl} 3$ to 5) events/h, 5 women) were studied. Subjects were imaged using T1-weighted 3-D structural MRI (69 subjects at 1.5 T, 51 subjects at $3 \mathrm{~T}$ ). Differences in grey matter were investigated in the two groups, controlling for age, sex, site and intracranial volume. Dedicated cerebellar analysis was performed on a subset of 108 scans using a spatially unbiased infratentorial template.

Results Patients with OSA had a reduction in grey matter volume in the right middle temporal gyrus compared with non-apnoeic controls $(p<0.05$, corrected for topological false discovery rate across the entire brain). A reduction in grey matter was also seen within the cerebellum, maximal in the left lobe VIIlb close to XI, extending across the midline into the right lobe.

Conclusion These data show that OSA is associated with focal loss of grey matter that could contribute to cognitive decline. Specifically, lesions in the cerebellum may result in both motor dysfunction and working memory deficits, with downstream negative consequences on tasks such as driving.

\section{INTRODUCTION}

Obstructive sleep apnoea (OSA) is a common disorder that causes intermittent hypoxia and frequent arousals from sleep, leading to extreme daytime sleepiness and increased cardiovascular risk. ${ }^{12}$ There is also a well-recognised link between OSA and cognitive impairment..$^{3-5}$ Specific impairments include reductions in working memory, phonological fluency, cognitive flexibility and planning; these can reduce efficiency at work, the ability to learn new tasks and remember instructions, ${ }^{6}$ and may underlie the increased road traffic accidents found in this population. ${ }^{7}$ Despite this link between OSA and cognitive impairment, little is known about neural deficits that may underlie these sequelae. Changes in neural activation associated with cognitive impairment ${ }^{8-13}$ and autonomic dysfunction ${ }^{14}$ have been recorded using functional MRI (fMRI) in patients with OSA; it is possible these functional changes are due to structural deficits.

Severe hypoxia and anoxia results in diffuse neurodegeneration ${ }^{15}$ whereas exposure to intermittent hypoxia has been shown to produce cellular damage within the CA1 region of the hippocampus and adjacent cortex in rats. ${ }^{16}$ In patients with OSA, several studies have used voxelbased morphometry (VBM) techniques to analyse structural magnetic resonance (MR) brain scans for changes in brain morphology. ${ }^{17-20}$ VBM enables voxel-wise comparisons of grey matter volume between two groups across the whole brain. ${ }^{21}$ Detection of a reduction in grey matter reflects neural damage or hypotrophy and is observed in a number of brain diseases, as well as normal ageing towards the latter part of life. ${ }^{22}$

Diffuse changes in grey matter concentration across the entire brain have been found in patients with OSA using VBM techniques, including the frontal, parietal and temporal cortices, anterior cingulate, hippocampus and cerebellum ${ }^{17}$ and bilateral prefrontal cortex, inferior parietal gyrus, right temporal and occipital cortices, right thalamus, some of the basal ganglia, right hippocampus and parahippocampal gyrus and cerebellum (right cerebellar hemisphere and vermis). ${ }^{20}$ However, these studies used a relatively low threshold for statistical significance $(p<0.001)$ uncorrected for multiple comparisons, ${ }^{17}$ or contained relatively few patients $(n=16+20)$. A smaller focal loss of grey matter within the left hippocampus was found in a very small group of patients with OSA $(n=7)$ using a level of significance selected on the basis of an a priori hypothesis. ${ }^{18}$ In contrast, a more stringent analysis $(p<0.05$, corrected for multiple comparisons using false discovery rate, FDR) showed no significant reductions in grey matter. ${ }^{19}$ All these studies had relatively small sample sizes (between 7 and 27 patients), they used different statistical thresholds and covariants, and some included patients with known comorbidities which might affect brain structure. This has resulted in confusion within the field. The present study aimed to determine whether OSA is associated with a focal loss of grey matter by studying a relatively large group of patients with OSA $(n=60)$ compared with a group of controls $(n=60)$ of 
similar age and gender using standardised improved methods of VBM analysis. Analyses of 60 of the 120 scans presented in this paper have been published elsewhere, although the analysis technique used has since been improved. ${ }^{18} 19$

\section{METHODS}

\section{Subjects}

Patients were recruited from sleep clinics in Melbourne, Australia (Department of Respiratory and Sleep Medicine, Austin Health) and London, UK (Royal Brompton and Charing Cross Hospitals). Patients with an apnoea/hypoponea index (AHI) $>30$ events/h were invited to participate in the study. The Melbourne patients also had to have at least $15 \%$ of total sleep time spent at oxygen saturation $\left(\mathrm{SaO}_{2}\right)<90 \%$. Exclusion criteria were a clinical diagnosis or history of respiratory disease, cerebrovascular or ischaemic heart disease, diabetes mellitus, central nervous system disorders (neurodegenerative diseases, epilepsy, head injury, psychosis, hypothyroidism, current depression), alcohol or illicit drug abuse, or current intake of psychoactive medications. Healthy controls were recruited by newspaper advertisement and through our database of healthy volunteers. In addition to the exclusion criteria above, the controls were also excluded from participation if they had a history of habitual snoring or any other sleep complaint. All control subjects were screened with polysomnography and questionnaire to exclude OSA.

Apnoeas were defined as $>80 \%$ drop in airflow for $\geq 10 \mathrm{~s}$ in the London dataset and a complete cessation of airflow for $\geq 10 \mathrm{~s}$ in the Melbourne data set. Hypopnoeas were defined as $>50 \%$ reduction in airflow from baseline with a $>4 \%$ dip in saturation, or an arousal from sleep in the London dataset, and $>50 \%$ decrease in airflow without a requirement for associated oxygen desaturation or arousal, plus those with a lesser airflow reduction in association with oxygen desaturation of $>3 \%$ or an arousal in the Melbourne dataset. Control subjects had an AHI $<5$ events $/ \mathrm{h}$ when airflow was measured by thermistor, or $<15$ events/h when airflow was measured by nasal cannula. ${ }^{23} 24$ The study was approved by the research and ethics committees in both institutions and all subjects gave informed written consent.

\section{MRI protocol}

Twenty-six patients with OSA and 25 healthy controls were studied in Melbourne, Australia. T1-weighted MR images were acquired using a $3.0 \mathrm{~T}$ scanner (General Electrics Horizon LX, Milwaukee, WI, USA). A fast spoilt, gradient-recalled echo at steady state sequence (time to repeat (TR) $13.8 \mathrm{~ms}$, time to echo (TE) $2.7 \mathrm{~ms}$, time to inversion (TI) $500 \mathrm{~ms}$, in-plane resolution $0.49 \times 0.49 \mathrm{~mm}$ ) with contiguous coronal slices of $2 \mathrm{~mm}$ thickness was used. Thirty-four patients with OSA and 35 healthy controls were studied in London, UK. T1-weighted MR images were acquired using a $1.5 \mathrm{~T}$ scanner (Magnetom Vision, Siemens Healthcare, Camberley, Surrey, UK) and a 3D MP-RAGE sequence (TI $300 \mathrm{~ms}$, TE $4 \mathrm{~ms}$, in-plane resolution $1.0 \times 1.0 \mathrm{~mm}$ ) with contiguous $2 \mathrm{~mm}$ coronal slices.

\section{Voxel-based morphometry}

VBM is an objective whole brain approach that enables comparisons of regional volumetric differences between groups. ${ }^{21}$ Briefly, VBM involves the segmentation of anatomical T1-weighted MR scans into grey matter, white matter and cerebrospinal fluid. The grey matter segments are then spatially normalised into standard stereotactic space using non-linear regression to align homologous regions across subjects. The resulting grey matter images are spatially smoothed to account for any remaining variation in gyral anatomy between individuals and to reduce spatial noise. Statistical analysis using the general linear model is performed to localise group differences using dedicated brain image analysis software (SPM; http:// www.fil.ion.ucl.ac.uk/spm). The 'optimised VBM' process used by previous publications in this field ${ }^{17-19}$ has now been improved by procedures within the current SPM software (SPM8) which provides higher quality spatial normalisation and segmentation; indeed, these steps are now combined (see below). In addition, SPM8 allows control of the FDR (a method used for controlling the false positive rate when performing multiple tests) of topological features (ie, clusters of voxels) which is more appropriate than controlling the FDR of individual voxels ${ }^{25}$ as was used in previous studies. ${ }^{19}$ The analysis output is produced in the form of a statistical parametric map (SPM) displaying voxels in which grey matter volume is significantly different from that which occurs in a control group.

In the present study the segmentation and normalisation steps were combined within a unified probabilistic framework provided in SPM8. ${ }^{26}$ In separate analyses the modulated grey matter segments were smoothed using either 8 or $12 \mathrm{~mm}$ Gaussian full-width-half-maximum smoothing kernels and saved at a voxel size of $2 \times 2 \times 2 \mathrm{~mm}$. The total intracranial volume was then calculated as the sum of the grey matter, white matter and cerebrospinal fluid segments over the whole brain.

\section{Spatially unbiased infratentorial template (SUIT) analysis}

There are intrinsic difficulties in the analysis of MR images of the cerebellum. ${ }^{27}$ In particular for VBM, standard spatial normalisation templates are optimised for the cortex and not for the cerebellum. These shortcomings in the standard template result in suboptimal grey matter segmentation of cerebellar tissues and potential inclusion of non-cerebellar tissue within the region of interest, ${ }^{27}$ factors that reduce accuracy and sensitivity. Furthermore, signal dropout can be relatively high in the cerebellum due to local field inhomogeneities. To address these issues we performed an additional analysis of the cerebellum using spatial segmentation and normalisation with a dedicated cerebellar template (spatially unbiased infratentorial template, SUIT 2.2). ${ }^{27}$ This template, based on the average anatomy of 20 healthy individuals, preserves the anatomical detail of the cerebellum better than the Montreal Neurological Institute whole brain template. The SUIT approach has been shown to increase the sensitivity of functional imaging studies which require accurate cerebellar normalisation by about $15 \% .{ }^{27}$

In brief, the MR image was masked using the cerebellum template before normalisation such that no supratentorial grey matter can bias the results. The subsequent normalisation and segmentation process used this cropped image and a classification map to create a non-linear deformation map matched to the SUIT template using the cosine-basis function approach. ${ }^{28}$ Finally, the images were resliced into the new atlas space and smoothed with $8 \mathrm{~mm}$ and $12 \mathrm{~mm}$ kernels.

\section{Statistical analysis}

Subject characteristics including age, body mass index (BMI) and sleep-disordered breathing parameters were tested using independent sample t tests to compare the patients with OSA and healthy controls.

Normalised (non-linearly registered) GM images were compared using a factorial design with site (London and Melbourne) and diagnosis (OSA patients and healthy controls) as factors. Age, sex and intracranial volume were included as covariates. By modelling the data in this way we were able to 
investigate the differences in grey matter volume between patients with OSA and healthy controls after adjusting for intersite differences in the imaging equipment and acquisition parameters.

Statistical parametric maps were first thresholded at $p<0.001$ uncorrected for multiple comparisons and then inferences about regionally specific grey matter volume differences were made using a significance threshold level of $p<0.05$ corrected for topological FDR across the whole brain. ${ }^{25}$ For the cerebellar analysis conducted as a post hoc comparison, the statistical threshold was set using a more stringent correction for multiple comparisons $(p<0.05$ family wise error $(F W E))$ across the cerebellum. These thresholds were chosen as the standard minimum acceptable to exclude excessive false positives. ${ }^{29}$ To assess the spatial homogeneity of the sensitivity to detect an effect, a sensitivity map was constructed that indicated the minimum change in grey matter volume required for each voxel to reach significance at $p<0.001$ uncorrected, following a variation of the method described in the appendix of the paper by Abbott et al. ${ }^{30}$ Specifically, rather than expressing sensitivity of each voxel as a percentage change in volume, we expressed sensitivity as an absolute change in volume in the same units as the contrast images (ie, we did not multiply by 100/local mean of the voxel).

The cerebellar SUIT analysis was conducted using a similar statistical model to that for the whole brain. Differences in overall cerebellar/brainstem volume were accounted for by including the combined grey and white matter volume of the segmented region as a covariate. One hundred and eight MR brain scans ( 52 patients with OSA, 56 healthy controls) were included in the SUIT analysis. Twelve scans (8 patients with OSA, 4 healthy controls) were excluded owing to poor cerebellar image quality or problems with segmentation in the cerebellum.

\section{RESULTS}

One hundred and twenty MR brain scans were analysed. Subject characteristics are given in table 1 . Sixty patients with severe OSA (26 recruited in Melbourne and 34 in London) and 60 healthy controls (25 in Melbourne and 35 in London) were studied. Overall, patients with OSA patients had a higher AHI, oxygen desaturation index, BMI and subjective sleepiness score (Epworth Sleepiness Scale, ESS) than healthy controls (all $p<0.001)$. There was no significant difference in the age between the two groups $(p=0.68)$.

Patients with OSA had a significant reduction in grey matter volume in the right middle temporal gyrus, extending along the occipito-temporal sulcus inferiorly $(\mathrm{p}<0.05$ corrected for topological FDR across the entire brain) compared with healthy controls (figure 1A). The outcome of the statistical analysis was similar for both the $8 \mathrm{~mm}$ and $12 \mathrm{~mm}$ smoothing kernels applied in preprocessing. The whole brain analysis also showed a significant reduction in cerebellar grey matter volume in a single bilateral region, with greatest extent and the voxel-wise statistical maximum within the left lobe (figure 1B). This finding was confirmed using the SUIT analysis, the location of the grey matter loss being within the left cerebellum lobe VIIIb but close to IX ( $p<0.05$ corrected for FWE across the cerebellum; figure 2).

The sensitivity analysis showed homogeneity in the ability to detect change throughout the brain, except for the brainstem and adjacent to the interhemispheric fissure (figure 3 ). The mean (SD) sensitivity within the regions of significant volume loss was 0.054 (0.007). To assess where in the brain the sensitivity was approximately as good or better than the regions in which there was a significant finding, we displayed the sensitivity map in pseudocolour such that cool colours (blue and green) indicate sensitivity as good or better than two standard deviations above the mean sensitivity of the regions of significant volume loss (ie, 0.067). Warm colours in the figure indicate areas where a larger volume change would be required before we could detect it in our study. This is about twice the sensitivity of our previous study. ${ }^{19}$ Maps showing the effect size associated with the change in grey matter are shown in figure 4. They indicate larger changes in the right temporal cortex and in the left cerebellum, changes that are consistent with the patterns of statistical significance.

\section{DISCUSSION}

Our study shows that regional grey matter loss does occur in patients with OSA. Using improved VBM analysis techniques in a relatively large dataset, we found a reduction in grey matter volume in the right temporal lobe and left cerebellum of patients with severe OSA. The functional consequences of these structural deficits remain to be determined.

Impact of different VBM analysis techniques on grey matter loss Our findings are consistent with previous studies ${ }^{17} 20$ which reported grey matter loss in the temporal lobe and cerebellum of patients with OSA. However, our data do not confirm previous reports of grey matter changes in the frontal and parietal cortices, anterior cingulate or the hippocampus, ${ }^{17} 1820$ areas important in autonomic regulation as well as cognitive function. The differences between the findings of the present study and those of previous studies cannot be explained by heterogeneity in sensitivity to detect reductions in grey matter volume in these regions. Our sensitivity map revealed relatively homogeneous sensitivity throughout most of the brain. The contradiction between the areas of grey matter volume reduction in our study and those of previous studies may therefore be explained by our use of improved image analysis techniques and appropriate statistical thresholds. It has been argued that the use of analysis

Table 1 Characteristics of study subjects

\begin{tabular}{|c|c|c|c|c|c|c|}
\hline \multirow[b]{3}{*}{ Group } & \multicolumn{3}{|l|}{ Patients with OSA } & \multicolumn{3}{|l|}{ Healthy controls } \\
\hline & \multicolumn{3}{|l|}{ Mean (95\% CI) } & \multicolumn{3}{|l|}{ Mean (95\% Cl) } \\
\hline & London $(n=34)$ & Melbourne $(n=26)$ & Total $(n=60)$ & London $(n=35)$ & Melbourne $(n=25)$ & Total $(n=60)$ \\
\hline BMI $\left(\mathrm{kg} / \mathrm{m}^{2}\right)^{*}$ & 31.4 (29.9 to 32.9 ) & 32.6 (31.0 to 34.3 ) & $32.0(30.9$ to 33.1$)$ & $24.7(23.4$ to 26.0$)$ & 25.3 (24.1 to 26.5$)$ & 25.0 (24.1 to 25.9$)$ \\
\hline $\mathrm{AHI}$ (events/h)* & 41.6 (33.2 to 49.9 ) & 71.5 (65.0 to 77.9$)$ & $55.0(48.3$ to 61.6$)$ & 2.1 (1.3 to 2.9$)$ & 5.3 (3.8 to 6.9$)$ & 4.1 (3.0 to 5.1) \\
\hline ODI (events/h)* & 33.0 (24.4 to 41.5 & - & $33.0(24.4$ to 41.5$)$ & $1.1(0.5$ to 1.7$)$ & - & $1.1(0.5$ to 1.7$)$ \\
\hline
\end{tabular}

*Significant difference between OSA patients and healthy controls $(\mathrm{p}<0.001)$. Normality was checked using SPSS 'normality plots.spv'. The plots appeared approximately normally distributed so independent sample $t$ test statistics were used to compare patients and controls.

$\mathrm{AHI}$, apnoea/hypopnoea index; BMI, body mass index; ESS, Epworth sleepiness scale; ODI, oxygen desaturation index; OSA, obstructive sleep apnoea. Bold type indicates data from all patients $(n=60)$, and all healthy controls $(n=60)$. 
A
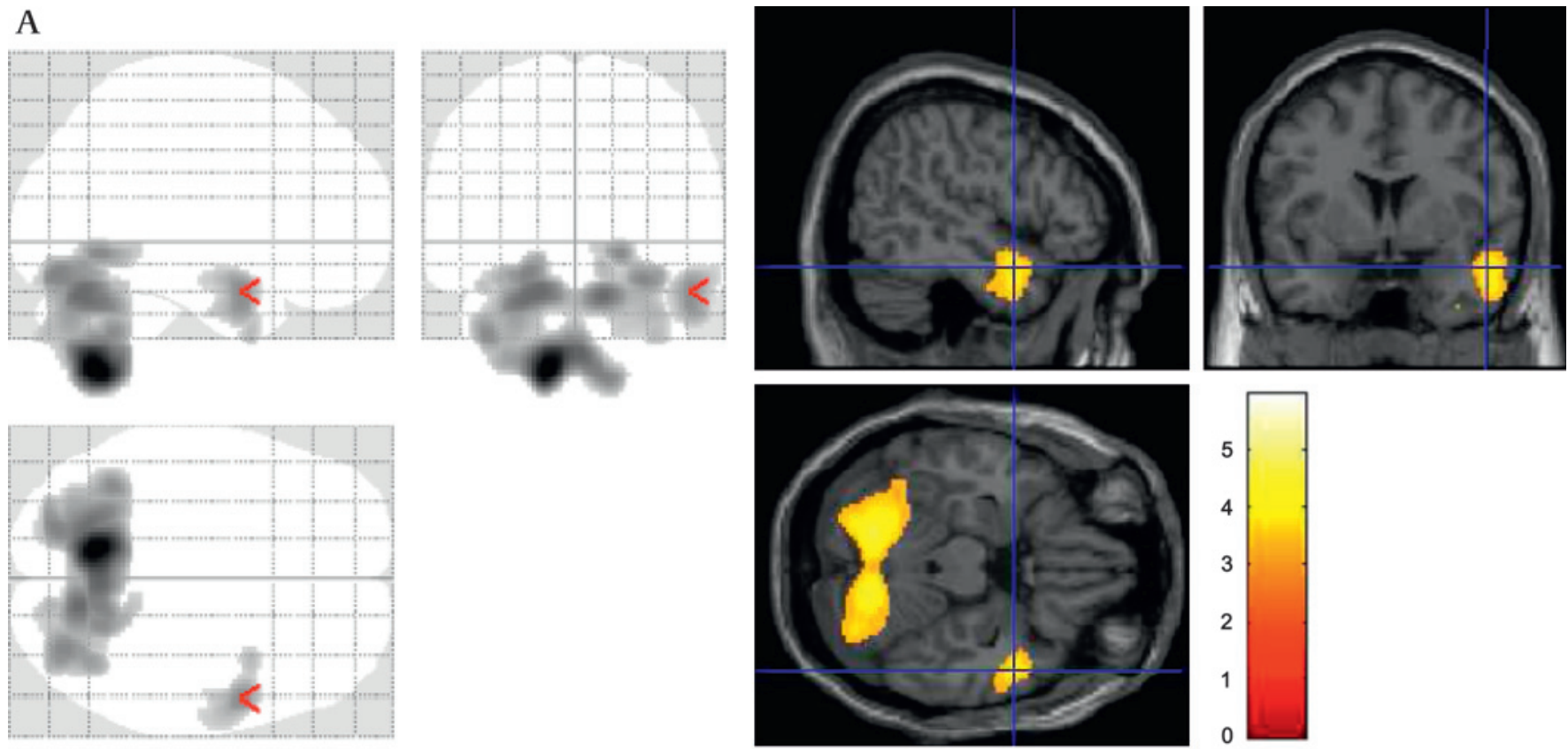

B
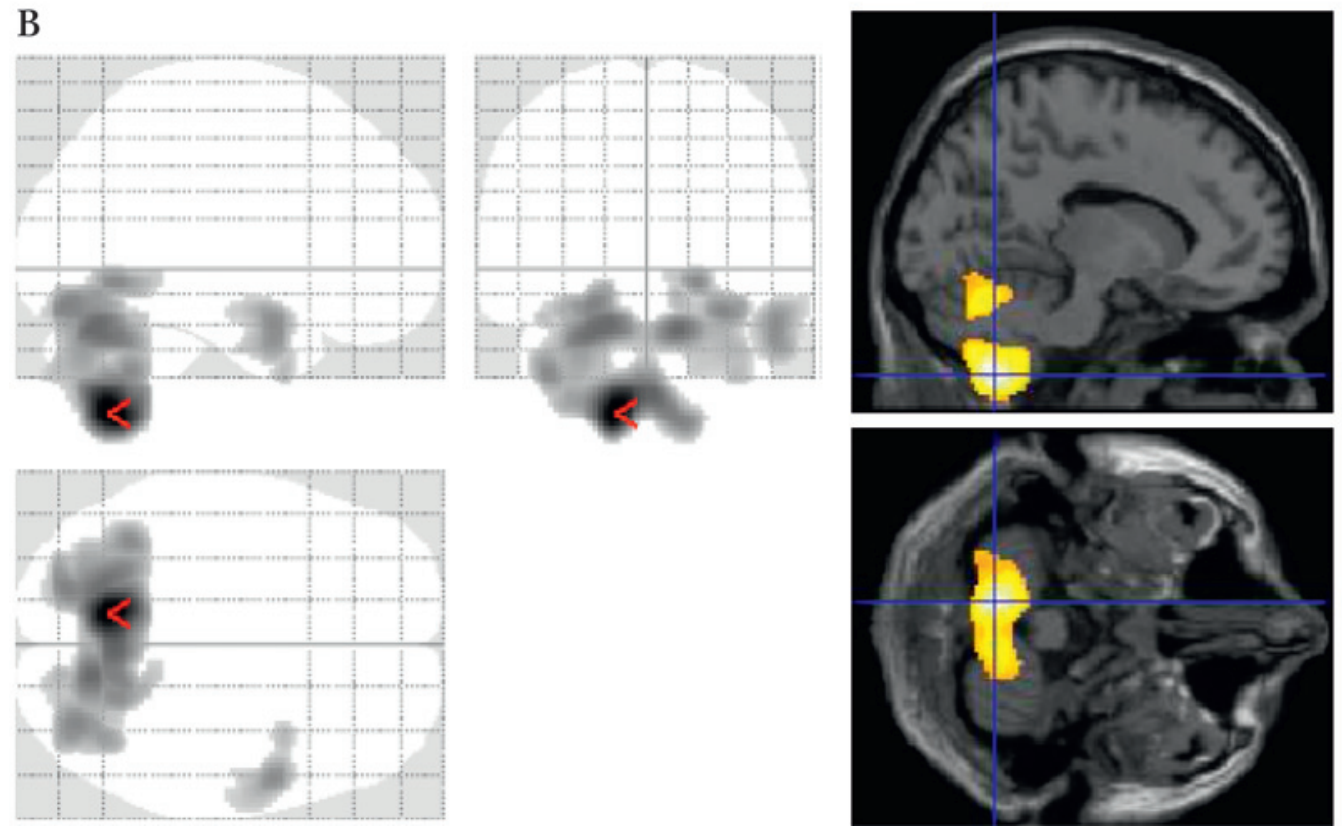

Figure 1 Images showing reductions in grey matter in patients with obstructive sleep apnoea (OSA) compared with controls. The left panel shows the 'glass brain' view and the right panel shows grey matter loss superimposed on an MR template. Views in neurological orientation (left is left) from top left clockwise are sagittal, coronal and transverse. (A) Reductions in grey matter in right middle temporal gyrus of patients with 0SA compared with controls. The voxel of maximum significance is marked with an arrowhead in the left panel. In the right panel the cross hairs ( $t=4.05$, indicated by a coloured bar) are located on the right at $x=52, y=4, z=-22 \mathrm{~mm}$ (Montreal Neurological Institute (MNI) coordinates relative to the anterior commissure). The images are thresholded to include clusters that survived the topological false detection rate threshold of $p<0.05$. (B) Reductions in grey matter in left cerebellum lobe VIIlb but close to IX in patients with OSA compared with controls. In the right panel the voxel of maximum significance $(t=5.95)$ is located on the left at $x=-12, y=-62, z=-56 \mathrm{~mm}$ (MNI). Note: the location of the cerebellum and the associated grey matter reduction extends below the standard glass brain reference grid.

thresholds that are too stringent will mask small differences in grey matter concentration between patients with OSA and controls. ${ }^{31}$ Nevertheless, some form of correction for multiple comparisons is required when using VBM techniques for whole brain comparisons to account for the very large number of voxels that make up the brain volume. ${ }^{19} 29$ Our level of inference differs from previous studies ${ }^{18}{ }^{19}$ in that we controlled for topological FDR across the entire brain. We chose this statistical threshold because lenient uncorrected thresholds are usually considered appropriate only for exploratory analyses, voxel-level FDR suffers from ambiguity in interpretation and voxel-level FWE can be too stringent if correcting across all brain voxels. Topological FDR provides a more appropriate level of control for type I and II errors when investigating where regions of grey matter loss occur across the brain. ${ }^{25}$

It has also been suggested that the magnitude of the changes in grey matter volume that occur in patients with OSA are similar to those seen with ageing, and that apnoea-related 
Figure 2 Images showing statistically significant reductions in cerebellar grey matter in a subset of patients with obstructive sleep apnoea (OSA) compared with controls using a spatially unbiased infratentorial template (SUIT): (A) 'glass brain' view; (B) superimposed on SUIT template. The image is displayed with a threshold of $p<0.001$ uncorrected. The voxel of maximum significance survives correction at $\mathrm{p}<0.05$ family wise error (few) corrected $(\mathrm{t}=4.62$, indicated by coloured bar) and is located on the left at $x=-14$, $y=-51, z=-48 \mathrm{~mm}$ (Montreal Neurological Institute (MNI) coordinates relative to the anterior commissure). Two further maxima were located on the inferior edge of the cerebellum ( $t=4.18$ : MNI: $-50,-58,-55 ; t=3.99$ : MNI: $-35,-53,-62)$. Note: the location of the cerebellum and the associated grey matter reduction extends below the standard glass brain reference grid.

changes may be missed if age is not included in an analysis of the covariance model. ${ }^{31}$ In both the present study and the previous study by O'Donoghue et al, ${ }^{19}$ age was included in the analysis of covariance, replicating this aspect of the analysis model of Macey et al. ${ }^{17}$ The use of the $12 \mathrm{~mm}$ smoothing kernel is also consistent across the present study and previous studies. ${ }^{17} 1820$

\section{Grey matter loss in the temporal lobe}

The decreased grey matter volume observed in the right middle temporal gyrus in patients with OSA is consistent with previous reports. ${ }^{17} 20$ These studies also found reductions in grey matter
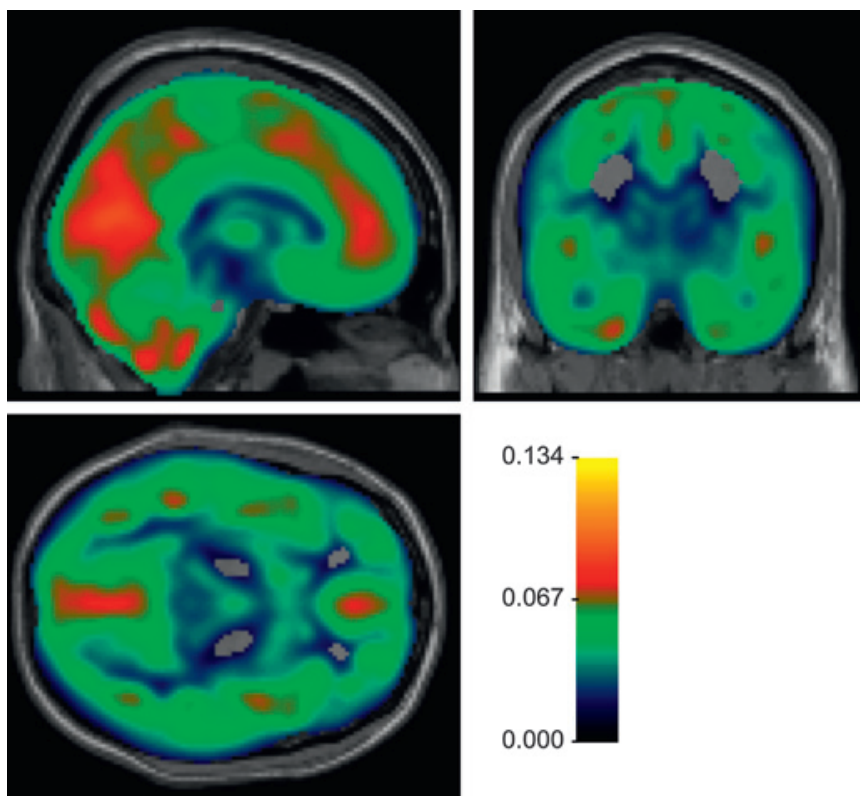

Figure 3 Sensitivity map of orthogonal planes passing through the anterior commissure (Montreal Neurological Institute 0, 0, 0). The map indicates the minimum change in grey matter volume required for each voxel to reach significance at $\mathrm{p}<0.001$ uncorrected (smaller is better). The mid point of the colour scale (0.067) is two standard deviations above the mean sensitivity of the voxels which were found to exhibit a significant difference between the groups (as illustrated in figure 1A, B). Cool colours (green and blue) indicate brain regions in which it would be expected that changes of approximately a similar magnitude could be detected, whereas warm colours indicate regions of relatively lower sensitivity. Sensitivity is good throughout most of the brain, except in the brainstem and interhemispheric fissure. in the inferior temporal gyrus, including the parahippocampa gyrus, and extending towards the anterior temporal pole. ${ }^{17} 20$ The precise function of the middle temporal gyrus is unclear. However, it is noteworthy that this area is susceptible to hypoxic damage in an animal model of OSA. ${ }^{16}$

Recent attempts to link the structural deficits in patients with OSA with functional consequences have concluded that structural deficits are associated with only minor memory and motor impairments. ${ }^{20}$ It would therefore appear that the structural deficits precede the functional consequences. Alternatively, only minor functional consequences may have been found if patients with OSA are able to compensate for structural deficits by increasing neuronal activation. Support for this suggestion is that brain activation is greater during verbal learning tasks performed by healthy people in a sleep-deprived state compared with a non-sleep deprived state ${ }^{32}$ and in patients with OSA compared with controls.'

\section{Grey matter loss in the cerebellum}

The whole brain analysis, controlling for topological FDR, suggested grey matter loss in a single large region of the cerebellum extending bilaterally but appearing to be more dominant on the left. As this was only a single region identified while controlling for topological FDR, it is difficult to be specific about the localisation of grey matter loss within the region. When thresholded at $\mathrm{p}<0.05$ corrected for FWE across the whole brain, the only portion of the region that survived was on the left. The issues associated with analysis of MR images of the cerebellum have been previously noted. ${ }^{27}$ Accordingly, we carried out our additional analysis using the SUIT technique to confirm the region of change. The SUIT analysis showed that the dominant region of grey matter loss was indeed lateralised to a limited area in the left cerebellum (figure 2).

The notion that OSA leads to cerebellar damage is intriguing. The cerebellum has a broad range of functions. Patients with right focal lesions often show verbal deficits whereas those with lesions on the left appear to suffer from more spatial deficits. ${ }^{33}$ In particular, the left VIIIB location has been highlighted as being involved in motor processing. ${ }^{34}$ Moreover, the links between the cerebellum and prefrontal activation suggest that working memory tasks which require a quick shift of attention may be impaired. ${ }^{35}$ Finally, cerebellum activation is increased during simulated driving. ${ }^{36}$ We speculate that impairments in attention and working memory, in association with excessive daytime sleepiness, could have negative consequences for daily 


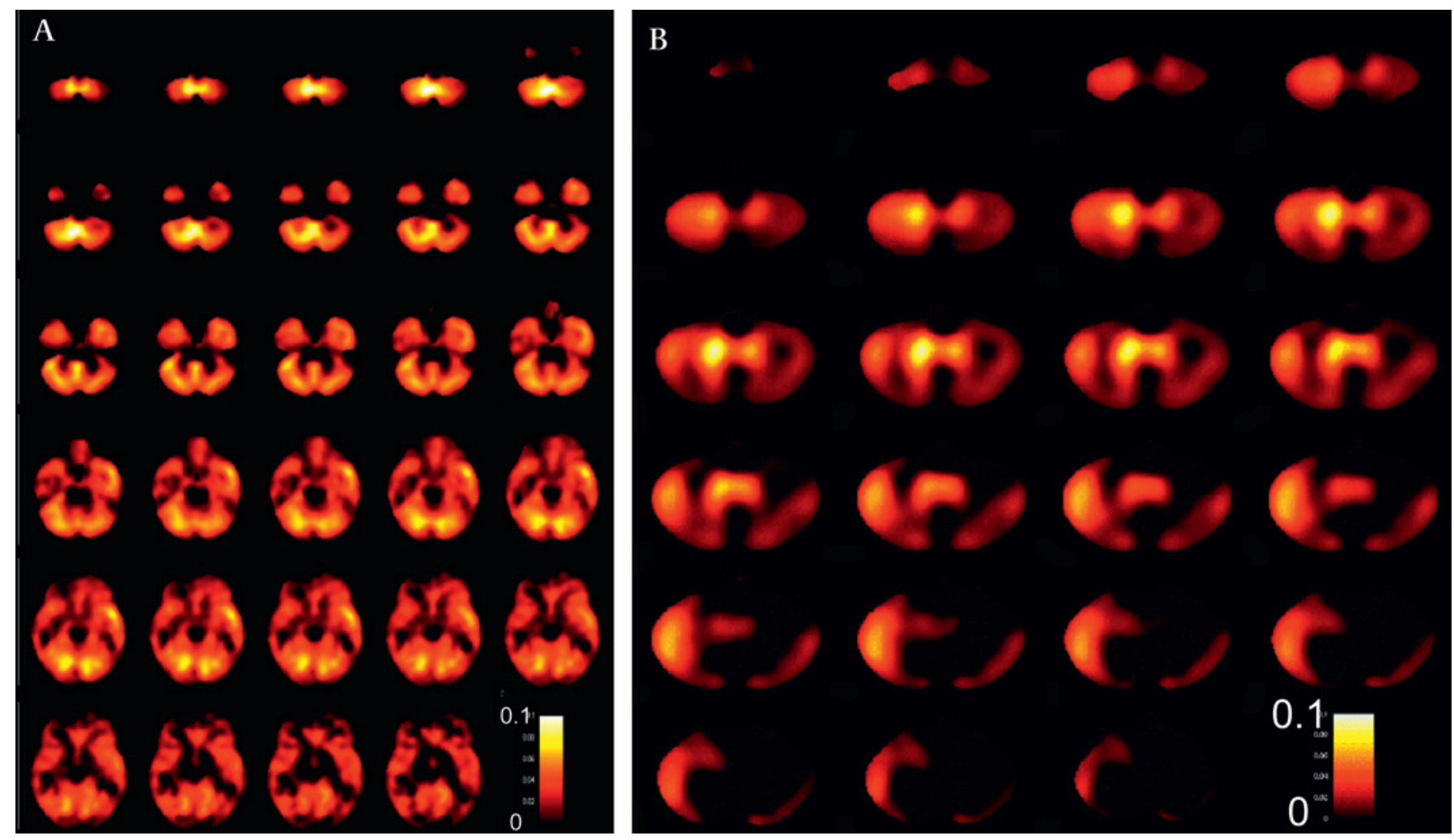

Figure 4 Size effect maps showing the magnitude of the differences in grey matter between controls and patients with obstructive sleep apnoea (OSA). (A) Whole brain analysis: changes from the base of the cerebellum $(z=-60 \mathrm{~mm})$ to a level immediately below the level of the anterior commissure $(\mathrm{z}=-4)$. (B) Cerebellum (SUIT) analysis. Each panel shows horizontal slices at $2 \mathrm{~mm}$ intervals. The colour scale indicates the size of the effects across the brain (hotter colours black to red to yellow to white indicate greater grey matter (fractional change in grey matter volume) in control versus OSA). These maps indicate larger changes in the right temporal cortex and in the left cerebellum; these changes are consistent with the patterns of statistical significance.

tasks carried out by patients with OSA and may in part explain the OSA-related increased risk of road traffic accidents. ${ }^{6}$ An alternative view is that neural deficits in the cerebellum may actually exacerbate or even cause sleep apnoea. ${ }^{37}$ Recent data which show a reduction in grey matter volume in the cerebellum of older people with previously undiagnosed sleep disordered breathing could support this idea ${ }^{38}$; whether such lesions precede the development of sleep apnoea is unknown.

\section{Other imaging modalities}

Other methods of MR analysis such as diffusion imaging enable early detection of structural changes in axons and their myelin sheaths. The axonal damage detected with diffusion MRI in the hippocampal, cerebellar and pontine neurons of patients with OSA may therefore occur before any reduction in grey matter is detectable with VBM. ${ }^{39}$ Reduced concentrations of N-acetylaspartate and choline in prefrontal white matter of patients with OSA compared with controls measured using magnetic resonance spectroscopy (MRS) would also support this idea of early structural (white matter) changes in patients with OSA. ${ }^{40}$ MRS changes have been found in the left hippocampus in a small number of patients with OSA compared with controls. Here the reduced creatine-containing compounds were correlated with OSA severity and cognitive impairment. ${ }^{41}$ These data suggest susceptibility of the hippocampal tissue to the intermittent hypoxia that occurs in OSA.

\section{Limitations}

A potential criticism of our study is that the acquisition of $M R$ images on two different scanners introduces a source of variance that may interfere with our ability to detect OSA-specific grey matter changes. Previous studies from one of our centres ${ }^{42} 43$ have shown that differences between scanners can be adequately controlled by incorporating site as a covariate in the statistical model. Our study is also well balanced across sites, so the results from one site are unlikely to dominate our SPM findings. However, to investigate this issue further we generated low threshold maps $(p<0.001)$ for both whole brain and cerebellum for each centre independently using SPM8 and a smoothing kernel of $12 \mathrm{~mm}$. This analysis showed that all changes were evident to some degree at low thresholds ( $p<0.001$ uncorrected) in both sets of data independently. The cerebellar changes were evident in both whole brain and dedicated cerebellum (at lenient thresholds) analysis.

It should be noted that the patients with OSA were heavier than the healthy controls and it is possible that neck fat may have degraded the MR scans at the level of the cerebellum. Studies in which the patients with OSA and controls are matched for BMI and neck size could test this issue, since the interrelationship between BMI and AHI makes it difficult to tease apart any effects statistically.

\section{Conclusions}

We found a reduction in grey matter volume in the right middle temporal gyrus and the left cerebellum in a large sample of patients with OSA compared with non-apnoeic healthy controls. These neural deficits could lead to impaired motor processing, divided attention tasks and working memory. We speculate that such deficits could, when linked with excessive daytime sleepiness, negatively affect everyday tasks such as driving. 
Acknowledgements Professor Rob Pierce died in tragic circumstances in the Victorian bushfires on 7 February 2009. His co-authors wish to dedicate this manuscript to his memory. We would like to thank Dr A K Simonds, Professor M I Polkey, Dr J Jamieson and $\operatorname{Dr} \mathrm{A}$ R C Cummin for allowing us to recruit patients from their clinics.

Funding The study was funded by The Wellcome Trust, Hammersmith Hospitals Trust, The National Heart and Lung Institute Foundation, The Australian National Health and Medical Research Council, Austin Health Medical Research Foundation, the Operational Infrastructure Support Program of the State government of Victoria, Australia, and ResMed Australia. It was supported by the NIHR Respiratory Disease Biomedical Research Unit at the Royal Brompton and Harefield NHS Foundation Trust and Imperial College London.

\section{Competing interests None.}

Ethics approval This study was conducted with the approval of the Royal Brompton Hospital.

Provenance and peer review Not commissioned; externally peer reviewed.

\section{REFERENCES}

1. Levy P, Pepin J-L, Arnaud C, et al. Intermittent hypoxia and sleep-disordered breathing: current concepts and perspectives. Eur Respir J 2008;32:1082-95.

2. Robinson GV, Stradling JR, Davies RJ. Sleep. 6: obstructive sleep apnoea/ hypopnoea syndrome and hypertension. Thorax 2004;59:1089-94.

3. Engleman HM, Douglas NJ. Sleep. 4: Sleepiness, cognitive function, and quality of life in obstructive sleep apnoea/hypopnoea syndrome. Thorax 2004:59:618-22.

4. Mazza S, Pepin J-L, Naegele B, et al. Most obstructive sleep apnoea patients exhibit vigilance and attention deficits on an extended battery of tests. Eur Respir $J$ 2005;25:75-80.

5. Twigg GL, Papaioannou I, Jackson ML, et al. Obstructive sleep apnoea syndrome is associated with deficits in verbal but not visual memory. Am J Respir Crit Care Med 2010:182:98-103.

6. McDaid C, Griffin S, Weatherly H, et al. Continuous positive airway pressure devices for the treatment of obstructive sleep apnoea-hypopnoea syndrome: a systematic review and economic analysis. Health Technol Assess 2009;13:1-142.

7. George CF. Sleep. 5: Driving and automobile crashes in patients with obstructive sleep apnoea/hypopnoea syndrome. Thorax 2004;59:804-7.

8. Thomas RJ, Rosen BR, Stern CE, et al. Functional imaging of working memory in obstructive sleep-disordered breathing. J Appl Physiol 2005;98:2226-34.

9. Ayalon L, Ancoli-Israel S, Klemfuss Z, et al. Increased brain activation during verbal learning in obstructive sleep apnea. Neuroimage 2006;31:1817-25.

10. Ayalon L, Ancoli-Israel S, Aka AA, et al. Relationship between obstructive sleep apnea severity and brain activation during a sustained attention task. Sleep 2009;32:373-81.

11. Ayalon L, Ancoli-Israel S, Drummond SP. Altered brain activation during response inhibition in obstructive sleep apnea. J Sleep Res 2009;18:204-8.

12. Archbold KH, Borghesani PR, Mahurin RK, et al. Neural activation patterns during working memory tasks and OSA disease severity: preliminary findings. J Clin Sleep Med 2009;5:21-7

13. Aloia MS, Sweet LH, Jerskey BA, et al. Treatment effects on brain activity during a working memory task in obstructive sleep apnea. J Sleep Res 2009:18:404-10.

14. Macey KE, Macey PM, Woo MA, et al. Inspiratory loading elicits aberrant fMRI signal changes in obstructive sleep apnea. Respir Physiol Neurobiol 2006:151:44-60.

15. Hopkins RO, Gale SD, Johnson SC, et al. Severe anoxia with and without concomitant brain atrophy and neuropsychological impairments. J Int Neuropsychol Soc 1995;1:501-9.

16. Gozal D, Daniel JM, Dohanich GP. Behavioural and anatomical correlates of chronic episodic hypoxia during sleep in the rat. J Neurosci 2001:21:2442-50.
17. Macey PM, Henderson LA, Macey KE, et al. Brain morphology associated with obstructive sleep apnea. Am J Respir Crit Care Med 2002;166:1382-7.

18. Morrell MJ, McRobbie DW, Quest RA, et al. Changes in brain morphology associated with obstructive sleep apnea. Sleep Med 2003:4:451-4.

19. O'Donoghue FJ, Briellmann RS, Rochford PD, et al. Cerebral structural changes in severe obstructive sleep apnea. Am J Respir Crit Care Med 2005:171:1185-90.

20. Yaouhi K, Bertran F, Clochon P, et al. A combined neuropsychological and brain imaging study of obstructive sleep apnea. J Sleep Res 2009;18:36-48.

21. Ashburner J, Friston KJ. Voxel-based morphometry-the methods. Neuroimage 2000;11:805-21.

22. May A, Gaser C. Magnetic resonance-based morphometry: a window into structural plasticity of the brain. Curr Opin Neurol 2006;19:407-11.

23. Series F, Marc I. Nasal pressure recording in the diagnosis of sleep apnoea hypopnoea syndrome. Thorax 1999;54:506-10.

24. Norman RG, Ahmed MM, Walsleben JA, et al. Detection of respiratory events during NPSG: nasal cannula/pressure sensor versus thermistor. Sleep 1997:20:1175-84.

25. Chumbley JR, Friston KJ. False discovery rate revisited: FDR and topological inference using Gaussian random fields. Neuroimage 2009;44:62-70.

26. Ashburner J, Friston KJ. Unified segmentation. Neuroimage 2005:26:839-51.

27. Diedrichsen J. A spatially unbiased atlas template of the human cerebellum. Neuroimage 2006;33:127-38.

28. Ashburner J, Friston KJ. Nonlinear spatial normalization using basis functions. Hum Brain Mapp 1999;7:254-66.

29. Ridgway GR, Henley SM, Rohrer JD, et al. Ten simple rules for reporting voxelbased morphometry studies. Neuroimage 2008;40:1429-35.

30. Abbott DF, Pell GS, Pardoe H, et al. Voxel-based iterative sensitivity (VBIS) analysis: methods and a validation of intensity scaling for T2-weighted imaging of hippocampa sclerosis. Neuroimage 2009;44:812-19.

31. Macey PM, Harper RM. OSA brain morphology differences: magnitude of loss approximates age-related effects. Am J Respir Crit Care Med 2005;172:1056-7: author reply 1057-8.

32. Drummond SP, Meloy MJ, Yanagi MA, et al. Compensatory recruitment after sleep deprivation and the relationship with performance. Psychiatry Res 2005; 140:211-23.

33. Gottwald B, Wilde B, Mihajlovic Z, et al. Evidence for distinct cognitive deficits after focal cerebellar lesions. J Neurol Neurosurg Psychiatry 2004;75:1524-31.

34. Stoodley CJ, Schmahmann JD. Functional topography in the human cerebellum: a meta-analysis of neuroimaging studies. Neuroimage 2009:44:489-501.

35. Schmahmann JD, Sherman JC. Cerebellar cognitive affective syndrome. Int Rev Neurobiol 1997;41:433-40.

36. Spires HJ, Maguire EA. Neural substrates of driving behaviour. Neuroimage 2007:36:245-55

37. Gozal D. The brain in sleep-disordered breathing: is it the chicken or is it the egg? Am J Respir Crit Care Med 2002:166:1305-6.

38. Celle S, Peyron R, Faillenot I, et al. Undiagnosed sleep-related breathing disorders are associated with focal brainstem atrophy in the elderly. Hum Brain Mapp 2009;30:2090-7

39. Macey PM, Kumar R, Woo MA, et al. Brain structural changes in obstructive sleep apnea. Sleep 2008:31:967-77.

40. Alchanatis $\mathbf{M}$, Deligiorgis N, Zias N, et al. Frontal brain lobe impairment in obstructive sleep apnoea: a proton MR spectroscopy study. Eur Respir $J$ 2004:24:980-6.

41. Bartlett DJ, Rae C, Thompson $\mathrm{CH}$, et al. Hippocampal area metabolites relate to severity and cognitive function in obstructive sleep apnea. Sleep Med 2004:5:593-6.

42. Pardoe H, Pell GS, Abbott DF, et al. Multi-site voxel-based morphometry: methods and a feasibility demonstration with childhood absence epilepsy. Neuroimage 2008:42:611-16.

43. Stonnington $\mathbf{C M}$, Tan G, Kloppel S, et al. Interpreting scan data acquired from multiple scanners: a study with Alzheimer's disease. Neuroimage 2008;39:1180-5. 